\title{
Incidence of Fractures among Epilepsy Patients: A Population-based Retrospective Cohort Study in the General Practice Research Database
}

\author{
*Patrick C. Souverein, †David J. Webb, †Hans Petri, †John Weil, †Tjeerd P. Van Staa, \\ and ${ }^{*}$ Toine Egberts \\ * Department of Pharmacoepidemiology and Pharmacotherapy, Utrecht Institute for Pharmaceutical Sciences, Utrecht University, \\ Utrecht, The Netherlands; †GlaxoSmithKline, WorldWide Epidemiology, New Frontiers Science Park, Harlow, and $\$$ Medical \\ Research Council, Environmental Epidemiology Unit, Southampton University Hospital, Southampton, England
}

Summary: Purpose: To compare the incidence of various fractures in a cohort of patients with epilepsy with a reference cohort of patients not having epilepsy.

Methods: Patients were included in the epilepsy cohort if they had at least one diagnosis of epilepsy in their medical history and had sufficient evidence of "active" epilepsy (use of antiepileptic drugs, diagnoses) after the practice was included in the General Practice Research Database (GPRD). Two reference patients were sampled for each patient with epilepsy from the same practice. Primary outcome was the occurrence of any fracture during follow-up. Poisson regression analysis was used to estimate incidence density ratios (IDRs).

Results: The study population comprised 40,485 and 80,970 patients in the epilepsy and reference cohorts, respectively. The median duration of follow-up was $\sim 3$ years. The overall inci- dence rate in the epilepsy cohort was 241.9 per 10,000 personyears. This rate was about twice as high as that in reference cohort: age- and sex-adjusted IDR, 1.89 (95\% CI, 1.81-1.98). When comparing IDRs among the different groups of fractures, the highest relative-risk estimate was found for hip and femur fractures (adjusted IDR, 2.79; 95\% CI, 2.41-3.24). IDRs were consistently elevated across age and sex groups and across fracture subtypes.

Conclusions: The overall risk of fractures was nearly twice as high among patients with epilepsy compared with the general population. The relative fracture risk was highest for hip and femur. Further study is necessary to elucidate whether this elevated risk is due to the disease, the use of antiepileptic drugs, or both. Key Words: Epilepsy-Incidence-FracturePopulation-based study—Cohort study_Epidemiology.
Epilepsy is a common chronic neurologic disease that requires long-term management and imposes a significant burden on health-care systems $(1,2)$. A meta-analysis by Kotsopoulos et al. (3) using data from 40 incidence studies showed a median incidence rate for epilepsy and unprovoked seizures of 47 per 100, 000 person-years. The incidence is highest in childhood and reaches a second peak in the elderly (60 years and older) (3).

Fractures are an important concern from a public health perspective, given their frequency and clinical consequences. It is difficult to establish the total cost associated with fractures. Most research has been conducted on hip fractures, as this type of fracture is one of the most serious fractures $(4,5)$. For hip fractures alone, it has been estimated that in the United Kingdom, the total cost to society is almost $£ 726$ million per annum (6).

Accepted September 24, 2004.

Address correspondence and reprint requests to Dr. P.C. Souverein at Department of Pharmacoepidemiology and Pharmacotherapy, Utrecht Institute for Pharmaceutical Sciences, P.O. Box 80082, 3508 TB Utrecht, The Netherlands. E-mail: P.C.Souverein@pharm.uu.nl
From the medical literature, it is known that patients with epilepsy have an increased risk of fractures compared with the general population (7). Some studies have found that this increased risk was related to seizures (8), whereas others found that the fracture risk was independent of seizure activity (9). In addition, use of antiepileptic drugs (AED) has emerged as an independent risk factor for fractures (10). The underlying mechanism may be an AED-induced decrease of bone mineral density, increasing fracture risk $(11,12)$, and/or sedative effects attributable to AEDs, leading to falls (13).

However, all these studies had either relatively small sample sizes, where not population-based, or had limited power to evaluate different fracture types. To our knowledge, the incidence of fractures among epilepsy patients has never been assessed in a large primarycare setting. Therefore the objective of this study was to compare the incidence of various fractures in a cohort of patients with epilepsy with a nonepilepsy reference cohort. 


\section{PATIENTS AND METHODS}

\section{Setting}

General practitioners (GPs) play a key role in the healthcare system in the United Kingdom, as they are responsible for primary health care and are gatekeepers for secondary care. The information in this study was obtained from the General Practice Research Database (GPRD), which contains the computerized medical records of $\sim 650$ general practices. Approximately 5 million of the total registered population of England and Wales is represented in the database. The data accrued in the GPRD include demographic information about the patient, diagnoses, prescription details, preventive care provided, referrals to specialist care, hospital admissions, and their major outcomes (14). Clinical data are stored and retrieved by means of Oxford Medical Information Systems (OXMIS) and Read codes for diseases or causes of morbidity and mortality that are cross-referenced to the International Classification of Diseases (ICD-9). Only data from practices that pass quality control are compiled to form the GPRD database. Several independent validation studies have shown that the GPRD database has a high level of completeness and validity (14). A validation study by Van Staa et al. (15) reported a high validity of the GPRD with respect to fractures. Hip fractures were confirmed by the GP on a questionnaire in $91.0 \%$ and vertebral fractures in $88.1 \%$ of fracture cases. However, vertebral fractures are often asymptomatic and might not appear in the GPRD. The GPRD is owned by the U.K. Department of Health and managed by the Medicines Control Agency (16). New software was introduced in GP practices in 1999, and no newer data were considered in this study, as the continuity of patient histories was lost since then.

\section{Study population}

The study period covered a period from January 1, 1990, to December 31, 1998. The source population comprised all permanently registered patients in the GRPD. All patients with at least one diagnosis relating to the presence of epilepsy in their medical records were identified. Patients were included in the epilepsy cohort if there was sufficient evidence in the medical records of "active" epilepsy. To assess whether the patient had such active epilepsy during GPRD follow-up, two scenarios were distinguished.

In the first scenario, patients had a diagnosis of epilepsy before the start of inclusion of the practice in the GPRD. This is possible because all practices are required to register all relevant medical events in the patients' medical history. However, when patients had a diagnosis of epilepsy in the past only, it could mean that they were symptom free at the time GPRD follow-up commenced. Therefore to be eligible for inclusion in the cohort, this category of patients was required to have at least one additional diagnosis of epilepsy or, alternatively, have a prescription for one of four main AEDs [carbamazepine (CBZ), sodium valproate (VPA), phenytoin (PHT), or phenobarbital (PB)] or ethosuximide (ESM) after the inclusion of the practice in the GPRD. Follow-up for these patients started at the first date of evidence for epilepsy after the inclusion of the practice in the GPRD (either the date of the epilepsy diagnosis or the prescription date). This date marked the start of follow-up. All patients with a diagnosis of epilepsy before the start of follow-up were classified having a nonspecified type of epilepsy, as the quality and continuity of this historical diagnosis were deemed to be uncertain.

In the second scenario, patients had a first diagnosis of epilepsy after the practice was included in the GPRD. "Active" epilepsy was assumed if at least one prescription for one of the five AEDs mentioned earlier was issued within 1 year after, but not on the date of the first epilepsy diagnosis, or if another diagnosis of epilepsy was registered within this period. Follow-up for these patients started at the date of the first epilepsy diagnosis. For all patients, the epilepsy status was monitored in yearly intervals. When a patient did not show any disease activity, as indicated by a prescription for any of the five drugs mentioned earlier or an epilepsy diagnosis in the year following the first 365 days after the start of follow-up, patients were censored. Furthermore, censoring occurred at the end of GPRD follow-up for the patient, at the first occurrence of a fracture during follow-up, or at the end of the study period.

The reference cohort included patients, randomly selected from the source population from those patients who did not have an epilepsy diagnosis at any point. To ensure active registration in the GPRD, all patients in the reference cohort had at least one entry in the GPRD after the start of GPRD follow-up. Two patients not having epilepsy were randomly sampled for each patient with epilepsy from the same GP practice and assigned the same follow-up start date.

\section{Outcome definition}

In the patients' medical records, the first occurrence of a fracture during GPRD follow-up was identified through relevant OXMIS en Read codes, which were subsequently converted to ICD-9 codes. The following classification scheme was applied: skull (ICD-9 categories 800-804), vertebra (805-806), rib (807), pelvis (808), clavicle (810), scapula (811), humerus (812), radius/ulna (813), hand (814-817), femur/hip (820/821), patella (822), tibia/fibula/ankle (823/824), foot (825/826), or unspecified fractures $(809,818,819,827-829)$. For practical purposes, fracture sites were categorized in a smaller number of groups: hip/femur, radius/ulna/hand, tibia/fibula/ankle/foot, and other fractures (including vertebral fractures).

\section{Data analysis}

All patients in the epilepsy and reference cohorts were monitored from the start of follow-up until the first 
occurrence of a fracture, the end of their registration in the GPRD, or the end of "active" (diagnoses/treatment) epilepsy. Incidence rates were calculated as the number of fractures divided by person-time. We stratified according to age (in three groups: younger than $20,20-49$, and 50 years or older) and sex. Crude incidence density ratios (IDRs) and 95\% confidence intervals (95\% CI) were calculated by dividing the incidence rate in the epilepsy cohort by the incidence rate in the reference cohort. Poisson regression analysis was used to estimate adjusted IDRs. Based on a background fracture incidence rate of $100 / 10,000$ person-years and a conservative assessment of the number of patients with epilepsy in the GPRD, a prestudy power calculation indicated that a sufficient number of patients with epilepsy would be available to detect a relative risk of 1.3 (alpha, 0.05; power, 0.95). Potential confounders evaluated in this study were history of fractures before GPRD follow-up and diagnosis of epilepsy before GPRD follow-up. Risk estimates were calculated for all fractures and for fracture subgroups. Data analysis was conducted with SAS, version 8.2 (Cary, NC, U.S.A.), and STATA, version 7.0 (College Station, TX, U.S.A.).

\section{RESULTS}

In the source population of $\sim 5$ million people, 59,279 patients with at least one epilepsy diagnosis and recorded age and gender details were identified. A total of 40,485 patients $(0.8 \%)$ met the definition for "active" epilepsy and were included in the epilepsy cohort. The reference cohort comprised 80,970 patients who did not have epilepsy. The characteristics of the epilepsy and reference cohorts are displayed in Table 1 . The median age was significantly higher in the epilepsy cohort compared with that in the reference cohort (39.1 vs. 34.1 years), whereas the distribution of males and females was similar. The median duration of follow-up was slightly higher in the reference cohort (3.3 vs. 3.0 years). The proportion of patients with a history of a fracture before the start of follow-up was higher among epilepsy patients than among patients not having epilepsy ( $11.7 \%$ and $7.6 \%$, respectively). Nearly $60 \%(n=23,791)$ of the patients in the epilepsy cohort had a first diagnosis of epilepsy before the practice started contributing to the GPRD, and for $43.0 \%$ of those patients, no further epilepsy diagnoses were registered after the practice was included in the GPRD. No difference was found in gender distribution between incident and prevalent patients with epilepsy, but incident patients with epilepsy were more often younger than 20 years at the start of follow-up (27.2\% vs. $13.3 \%$ ), whereas the prevalent patients with epilepsy were more frequently aged between 20 and 59 years ( $45.6 \%$ vs. $61.4 \%$ ), respectively. The majority of the epilepsy diagnoses in the GP's records were not specified (89.3\%; e.g., epilepsy convulsions and epileptic

TABLE 1. Characteristics of the epilepsy $(n=40,485)$ and control cohort $(n=80,970)$

\begin{tabular}{|c|c|c|c|c|}
\hline \multirow{4}{*}{$\begin{array}{l}\text { Characteristic } \\
\text { Age (median) } \\
\text { Age (years) }\end{array}$} & \multicolumn{2}{|c|}{$\begin{array}{l}\text { Epilepsy cohort } \\
(\mathrm{n}=40,485)\end{array}$} & \multicolumn{2}{|c|}{$\begin{array}{l}\text { Reference cohort } \\
\quad(\mathrm{n}=80,970)\end{array}$} \\
\hline & No. & $(\%)$ & No. & $(\%)$ \\
\hline & \multicolumn{2}{|c|}{$39.1 \mathrm{yrs}$} & \multicolumn{2}{|c|}{$34.1 \mathrm{yrs}$} \\
\hline & & & & \\
\hline$<20$ & 7,695 & $(19.0)$ & 20,745 & (25.6) \\
\hline $20-49$ & 18,185 & $(44.9)$ & 36,105 & $(44.6)$ \\
\hline$\geq 50$ & 14,605 & $(36.1)$ & 24,120 & $(29.8)$ \\
\hline \multicolumn{5}{|l|}{ Gender } \\
\hline Male & 20,252 & $(50.0)$ & 38,224 & $(47.2)$ \\
\hline Female & 20,233 & $(50.0)$ & 42,746 & $(52.8)$ \\
\hline Median duration of follow-up & $3.0 \mathrm{yrs}$ & $3.3 \mathrm{yrs}$ & & \\
\hline History of fractures before cohort entry & 4,749 & $(11.7)$ & 6,162 & $(7.6)$ \\
\hline Diagnosis of epilepsy before practice up to standard & 23,791 & $(58.8)$ & & \\
\hline \multicolumn{5}{|l|}{ Type of epilepsy $*$} \\
\hline Nonspecific & 36,153 & $(89.3)$ & & \\
\hline Partial epilepsy & 1,379 & $(3.4)$ & & \\
\hline - Temporal lobe epilepsy & 976 & $(2.4)$ & & \\
\hline - Jacksonian Seizure & 370 & $(0.9)$ & & \\
\hline - Other/unspecified partial epilepsy & 33 & $(0.1)$ & & \\
\hline Generalized epilepsy & 2,631 & $(6.5)$ & & \\
\hline - Absence (petit mal) & 1,059 & $(2.6)$ & & \\
\hline - Tonic-clonic (grand mal) & 1,509 & $(3.7)$ & & \\
\hline - Other/unspecified generalized epilepsy & 63 & $(0.2)$ & & \\
\hline Status epilepticus & 322 & $(0.8)$ & & \\
\hline
\end{tabular}

*First diagnosis of epilepsy after the start of GPRD qiuality up to date; if patients had a diagnosis of epilepsy prior to the start of GPRD follow-up only, the type of epilepsy was classified as nonspecific. 
TABLE 2. Incidence rates of fractures in the epilepsy and reference cohort

\begin{tabular}{|c|c|c|c|c|c|}
\hline Type of fracture & Number of fractures & Person-years & Rate/10,000 person-years & Crude IRR (95\% CI) & Adjusted IRR(95\% CI) \\
\hline \multicolumn{6}{|l|}{ Any fracture } \\
\hline Control & 3,940 & 319,142 & 123.5 & 1.00 (Reference) & 1.00 (Reference) \\
\hline Epilepsy & 3,478 & 143,754 & 241.9 & $1.96(1.87-2.05)$ & $1.89(1.81-1.98)$ \\
\hline \multicolumn{6}{|c|}{ Hip/femur fracture } \\
\hline Control & 301 & 319,142 & 9.4 & 1.00 (Reference) & 1.00 (Reference) \\
\hline Epilepsy & 436 & 143,754 & 30.3 & $3.22(2.78-3.72)$ & $2.79(2.41-3.24)$ \\
\hline \multicolumn{6}{|c|}{ Hand or radius/ulna fracture } \\
\hline Control & 1,445 & 319,142 & 45.3 & 1.00 (Reference) & 1.00 (Reference) \\
\hline Epilepsy & 1,101 & 143,754 & 76.7 & $1.69(1.56-1.83)$ & $1.70(1.57-1.84)$ \\
\hline \multicolumn{6}{|c|}{ Tibia, fibula, ankle or foot fracture } \\
\hline Control & 749 & 319,142 & 23.5 & 1.00 (Reference) & 1.00 (Reference) \\
\hline Epilepsy & 640 & 143,754 & 44.5 & $1.90(1.71-2.11)$ & $1.89(1.70-2.10)$ \\
\hline \multicolumn{6}{|l|}{ Other fractures } \\
\hline Control & 1,491 & 319,142 & 46.7 & 1.00 (Reference) & 1.00 (Reference) \\
\hline Epilepsy & 1,351 & 143,754 & 94.0 & $2.01(1.87-2.17)$ & $1.94(1.80-2.09)$ \\
\hline
\end{tabular}

IRR, Incidence rate ratio, CI; Confidence interval,

*Adjusted for age and sex.

Patients can have multiple fractures sites.

fit). Temporal lobe epilepsy was the most commonly diagnosed form of partial epilepsy (2.4\%), whereas absences and tonic-clonic seizures were the most common types of generalized epilepsy.

Table 2 shows the overall incidence rates of fractures in the epilepsy and the reference cohort. In the epilepsy cohort, 3,489 fractures occurred during 143,754 personyears of follow-up, yielding an overall incidence rate of 241.9 per 10,000 person-years. This rate was about twice the incidence rate of the reference cohort $(123.5 / 10,000$ person-years). The crude IDR was 1.96 (95\% CI, 1.872.05), and decreased slightly to 1.89 (1.81-1.98) after adjustment for age and sex. Adjustment for history of fractures did not affect risk estimates.

IDRs were consistently elevated across age and sex groups and across fracture subtypes. The highest risk estimate was found for hip and femur fractures (adjusted IDR, 2.79; 95\% CI, 2.41-3.24). The adjusted IDRs for fractures of the hand and arm, lower leg and foot, and other fractures were 1.70 (95\% CI, 1.57-1.84), 1.89 (95\% CI, 1.70-2.10), and 1.94 (95\% CI, 1.80-2.09), respectively. Figure 1 shows the age group-specific fracture incidence rates for all and site-specific fractures, stratified by sex. The corresponding IDR estimates are displayed in Table 3. For all fractures, the incidence rates in both the epilepsy and the reference cohort were higher in male than in female patients in the age categories younger than 50 years. However, whereas the incidence decreased with age among male patients, there was a sharp increase among women aged 50 years and older. The corresponding IDRs increased with age from 1.37 to 2.24 for male and 1.43 to 2.34 for female subjects, which is relevant, as the majority of fractures occurred in the highest age group. Hip and femur fractures were infrequent among patients younger than 50 years, but a large difference in incidence between those with and without epilepsy was observed in the old- est age category. The IDRs in the younger than 50 year group among male and female patients were 3.07 (95\% CI, 2.32-4.08) and 2.75 (95\% CI, 2.30-3.29), respectively. Fractures of the radius, ulna, and hand were particularly common among boys younger than 20 years (incidence rates, $138.4 / 10,000$ person-years in the epilepsy cohort and 95.1/10,000 person-years in the reference cohort). Among male patients, the incidence of this type of fracture decreased with age, whereas it increased in women older than 50 years. The largest differences between the epilepsy and control cohorts were in the highest age group, with risk estimates in both sexes $\sim 2.2$. The incidence rates of foot, ankle, tibia, and fibula fractures decreased with age among male, but increased among female patients. The point estimates of the IDR slightly increased with age from 1.54 to 1.75 (male) and 1.96 to 2.23 (female patients). For other fractures (including vertebral fractures), the similar incidence increase among female subjects was observed. The IDRs were $\sim 1.3$ for male and female patients younger than 20 years, and were around 2.3 in the middle and high age groups for both sexes.

Table 4 shows the incidence rates and IDRs after stratification on timing of the first epilepsy diagnosis. Patients who had a first diagnosis of epilepsy after the start of GPRD follow-up had a higher risk of fractures compared with patients having a diagnosis before the start of GPRD follow-up (adjusted IDR, 2.12; 95\% CI, 1.95-2.30; and $1.80,95 \%$ CI, 1.70-1.90, respectively). These findings were similar for all fracture subtypes, except for fractures of the foot, ankle, tibia, and fibula (see Table 4).

\section{DISCUSSION}

In this population-based study, we found that the risk of fractures among patients with epilepsy was approximately twofold higher compared with general population 

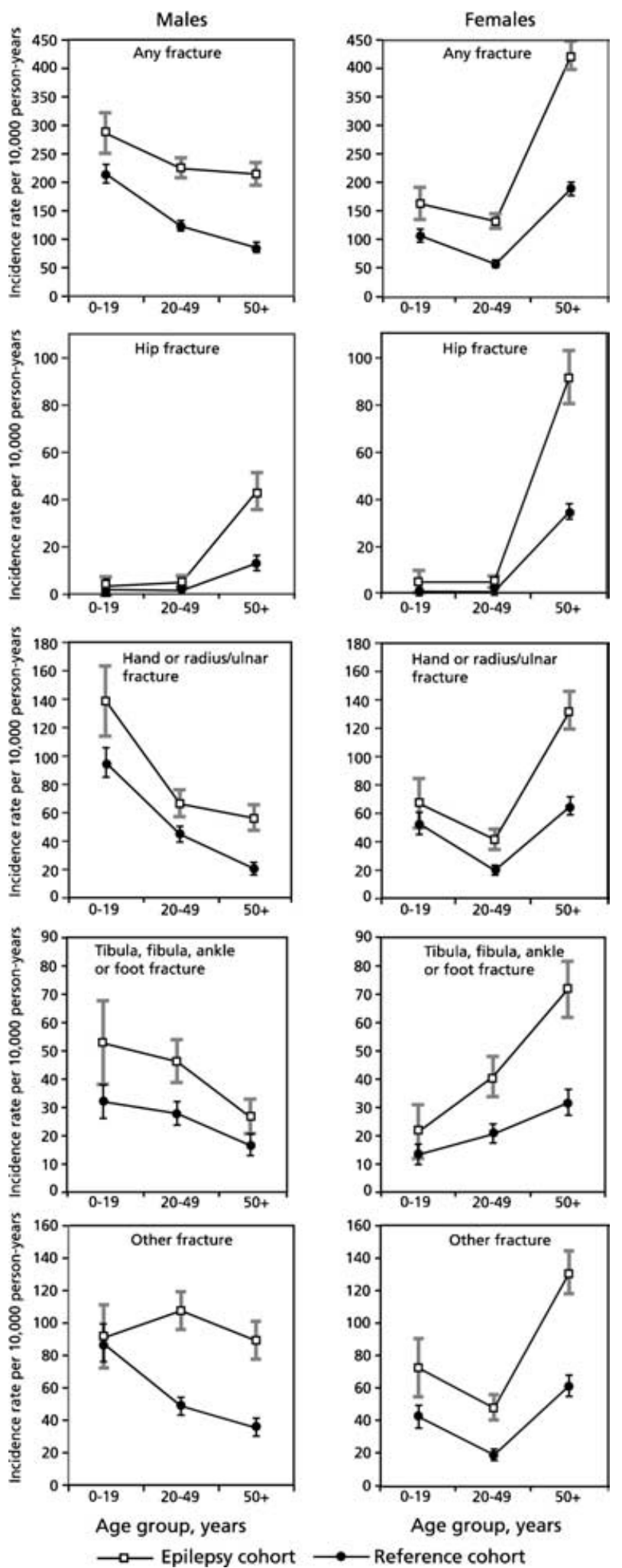

FIG. 1. Incidence rates of fractures in the epilepsy and reference cohort by age category and sex. patients not having epilepsy. IDRs were consistently elevated across age and sex groups and across fracture subtypes. Of particular clinical relevance is our finding that the risk of hip fractures was threefold increased in both men and women aged 50 years and older. The fracture incidence rates found in the reference cohort resemble the rates reported by Van Staa et al. (17) in a large study on the incidence of fractures in England and Wales.

A strength of this study is the large sample size, as we studied $>40,000$ patients with epilepsy, enabling us to look at different types of fractures. In the medical literature, other studies investigated incidences of fractures in noninstitutionalized patients with epilepsy. The limitation of these studies was either a relatively small sample size, with the consequence that fracture-specific incidences or risk estimates often could not be provided because of lack of statistical power, or the fact that data were obtained through interviews and questionnaires rather than routinely collected medical data, resulting in possible biases due to selective nonresponse and recall problems, or both. Annegers et al. (18) determined whether the number of observed fractures in a cohort of 467 patients with unprovoked seizures in Rochester, Minnesota, was higher than expected. Standardized morbidity ratios were significantly increased for hip fracture, but not for distal forearm fracture. Vestergaard et al. (8) found that the relative risk of fractures was $2.0(95 \%$ CI, 1.6-2.5) in a Danish historical follow-up study comparing fracture incidence among 345 epilepsy patients and 645 controls. Information on fractures was obtained via questionnaires. Persson et al. (7) found that the incidence of extremity fractures among 177 epilepsy patients was $23.8 / 1,000$ person-years of observation, higher than expected (SMR, 2.39; 95\% CI, 1.52-3.59) (7). The highest SMRs were found for men aged 45 years and older, and in particular, for combined hip, patellar, tibia, fibula, and foot fractures (7). However, the number of patients having an extremity fracture in this study was low $(n=20)$, and statistical power is therefore low. Cummings et al. (10) identified use of AEDs as an independent risk factor for hip fracture in a cohort study of 9,516 white female subjects. Monitoring of hip-fracture occurrence was done by telephone interviews and postcards. As the study did not focus on epilepsy, the number of hip fractures among current users of AEDs totalled just seven, and none of these fractures resulted from seizures and loss of consciousness. Despite these articles, Mattson and Gidal (19) noticed in a recent review that no prospective studies defined the frequency of fractures in patients with epilepsy. Therefore the results of our study appear to add on existing knowledge.

The main limitation of the study was that no opportunity was found to interview patients directly on their epilepsy status. To minimize the possibility of diluting the contrast between the epilepsy and reference cohorts, a rather strict definition for inclusion in the epilepsy cohort was 
TABLE 3. Incidence Rate Ratios (IRR) for epilepsy versus reference cohort stratified by sex and age group

\begin{tabular}{|c|c|c|c|c|}
\hline \multirow[b]{2}{*}{ Type of fracture } & \multicolumn{2}{|c|}{ Men } & \multicolumn{2}{|c|}{ Women } \\
\hline & $\#$ events $^{a}$ & IRR $(95 \%$ CI $)$ & $\#$ events $^{a}$ & IRR $(95 \%$ CI $)$ \\
\hline \multicolumn{5}{|l|}{ Any fracture } \\
\hline Age $<20$ & $266 / 726$ & $1.37(1.22-1.55)$ & $145 / 367$ & $1.43(1.26-1.64)$ \\
\hline Age $20-49$ & $742 / 814$ & $1.95(1.79-2.13)$ & $445 / 410$ & $2.04(1.84-2.26)$ \\
\hline Age $\geq 50$ & $631 / 437$ & $2.24(2.04-2.47)$ & $1,249 / 1,186$ & $2.34(2.18-2.52)$ \\
\hline \multicolumn{5}{|c|}{ Hip/femur fracture } \\
\hline Age $<20$ & $3 / 6$ & $3.40(1.22-9.47)$ & $4 / 2$ & $3.05(1.08-8.57)$ \\
\hline Age $20-49$ & $16 / 9$ & $5.56(2.81-10.99)$ & $15 / 3$ & $4.98(2.49-9.94)$ \\
\hline Age $\geq 50$ & $128 / 67$ & $3.07(2.32-4.08)$ & $270 / 214$ & $2.75(2.30-3.29)$ \\
\hline \multicolumn{5}{|c|}{ Hand or radius/ulnar fracture } \\
\hline Age $<20$ & $129 / 322$ & $1.40(1.17-1.67)$ & $59 / 179$ & $1.37(1.12-1.68)$ \\
\hline Age $20-49$ & $219 / 297$ & $1.69(1.45-1.97)$ & $138 / 138$ & $1.65(1.38-1.98)$ \\
\hline Age $\geq 50$ & $165 / 107$ & $2.23(1.87-2.68)$ & $391 / 401$ & $2.19(1.93-2.49)$ \\
\hline \multicolumn{5}{|c|}{ Tibia, fibula, ankle or foot fracture } \\
\hline Age $<20$ & $49 / 108$ & $1.54(1.15-2.07)$ & $18 / 42$ & $1.96(1.41-2.73)$ \\
\hline Age $20-49$ & $153 / 185$ & $1.63(1.35-1.97)$ & $133 / 138$ & $2.08(1.70-2.54)$ \\
\hline Age $\geq 50$ & $78 / 85$ & $1.75(1.39-2.21)$ & $209 / 191$ & $2.23(1.86-2.67)$ \\
\hline \multicolumn{5}{|l|}{ Other fracture } \\
\hline Age $<20$ & $89 / 297$ & $1.27(1.05-1.54)$ & $66 / 149$ & $1.32(1.07-1.63)$ \\
\hline Age $20-49$ & $361 / 325$ & $2.29(2.01-2.62)$ & $160 / 133$ & $2.38(2.01-2.82)$ \\
\hline Age $\geq 50$ & $272 / 186$ & $2.22(1.91-2.58)$ & $403 / 401$ & $2.30(2.03-2.61)$ \\
\hline
\end{tabular}

${ }^{a}$ Epilepsy/control group, respectively.

IRR, Incidence rate ratio, CI; Confidence interval.

applied. Patients were required to show disease activity, either by having prescriptions for AEDs or, alternatively, by having diagnoses of epilepsy. The criteria used might have resulted in an overrepresentation of patients having more severe epilepsy in our cohort and, as patients with more severe epilepsy are arguably more at risk of falling and fracturing compared with patients with lower disease severity, an overestimation of IDRs. We were not able to validate epilepsy diagnoses in this study. For our defini- tion, only medical codes with a specific mentioning of epilepsy were included. Diagnoses of "fit" were not considered to be sufficient, whereas a diagnosis of "epileptic fit" was. Furthermore, only the main five AEDs were evaluated to monitor epilepsy activity, although many more are available on the market. This could have resulted in the exclusion or premature censoring of eligible epilepsy patients using other AEDs. However, the drugs that were chosen were the main AEDs used in epilepsy treatment,

TABLE 4. Incidence rates of fractures in the epilepsy and reference cohort by timing of first diagnosis of epilepsy

\begin{tabular}{|c|c|c|c|c|c|c|c|c|}
\hline \multirow[b]{2}{*}{$\begin{array}{l}\text { Type of } \\
\text { fracture }\end{array}$} & \multicolumn{4}{|c|}{$\begin{array}{l}\text { First diagnosis of epilepsy } \\
\text { during GPRD follow-up }\end{array}$} & \multicolumn{4}{|c|}{$\begin{array}{l}\text { First diagnosis of epilepsy } \\
\text { before GPRD follow-up }\end{array}$} \\
\hline & $\begin{array}{l}\text { Number of } \\
\text { fractures }\end{array}$ & $\begin{array}{l}\text { Rate/10,000 } \\
\text { person-years }\end{array}$ & $\begin{array}{c}\text { Crude IRR } \\
(95 \% \mathrm{CI})\end{array}$ & $\begin{array}{l}\text { Adjusted IRR } \\
\quad(95 \% \mathrm{CI})\end{array}$ & $\begin{array}{l}\text { Number of } \\
\text { fractures }\end{array}$ & $\begin{array}{l}\text { Rate/10,000 } \\
\text { person-years }\end{array}$ & $\begin{array}{l}\text { Crude IRR } \\
(95 \% \mathrm{CI})\end{array}$ & $\begin{array}{l}\text { Adjusted IRR } \\
\quad(95 \% \mathrm{CI})\end{array}$ \\
\hline \multicolumn{9}{|l|}{ Any fracture } \\
\hline Control & 1,207 & 118.5 & 1.00 (Reference) & 1.00 (Reference) & 2,733 & 125.8 & 1.00 (Reference) & 1.00 (Reference) \\
\hline Epilepsy & 1,102 & 257.1 & $2.17(2.00-2.35)$ & $2.12(1.95-2.30)$ & 2,376 & 235.5 & $1.87(1.77-1.98)$ & $1.80(1.70-1.90)$ \\
\hline \multicolumn{9}{|c|}{ Hip/femur fracture } \\
\hline Control & 95 & 9.3 & 1.00 (Reference) & 1.00 (Reference) & 206 & 9.5 & 1.00 (Reference) & 1.00 (Reference) \\
\hline Epilepsy & 164 & 38.3 & $4.10(3.17-5.34)$ & $3.31(2.57-4.27)$ & 272 & 27.0 & $2.84(2.36-3.42)$ & $2.58(2.15-3.09)$ \\
\hline \multicolumn{9}{|c|}{$\begin{array}{l}\text { Hand or radius/ } \\
\text { ulna fracture }\end{array}$} \\
\hline Control & 435 & 42.7 & 1.00 (Reference) & 1.00 (Reference) & 1,010 & 46.5 & 1.00 (Reference) & 1.00 (Reference) \\
\hline Epilepsy & 321 & 74.9 & $1.75(1.51-2.03)$ & $1.76(1.53-2.04)$ & 780 & 77.3 & $1.66(1.51-1.83)$ & $1.66(1.51-1.83)$ \\
\hline \multicolumn{9}{|c|}{$\begin{array}{l}\text { Tibia, fibula, ankle } \\
\text { or foot fracture }\end{array}$} \\
\hline Control & 250 & 24.5 & 1.00 (Reference) & 1.00 (Reference) & 499 & 23.0 & 1.00 (Reference) & 1.00 (Reference) \\
\hline Epilepsy & 172 & 40.1 & $1.64(1.34-1.99)$ & $1.63(1.34-1.97)$ & 468 & 46.4 & $2.02(1.78-2.30)$ & $2.02(1.77-2.29)$ \\
\hline \multicolumn{9}{|c|}{ Other fracture } \\
\hline Control & 440 & 43.2 & 1.00 (Reference) & 1.00 (Reference) & 1,051 & 48.3 & 1.00 (Reference) & 1.00 (Reference) \\
\hline Epilepsy & 462 & 107.8 & $2.50(2.19-2.85)$ & $2.43(2.13-2.77)$ & 889 & 88.1 & $1.82(1.66-1.99)$ & $1.75(1.60-1.92)$ \\
\hline
\end{tabular}

IRR, Incidence rate ratio; CI, Confidence interval, adjusted for age and sex.

Patients can have multiple fractures sites. 
and the market share of other AEDs in the United Kingdom was relatively small during the study period. An exploratory analysis of all patients using AEDs in our cohort showed that $99 \%$ used one of these five AEDs. Misclassification of epilepsy cannot be ruled out, given the nature of our epilepsy definition. All patients in the reference cohort did not have a diagnosis of epilepsy at any stage. However, they could have used AEDs, either for other indications or because a diagnosis was not entered by the patients' GP. Because such misclassification is likely to be nondifferential, it will have diluted the true risk estimates.

Patients with a history of fractures are known to be at a higher risk of subsequent fractures. It also is possible that patients with a history of epilepsy before the inclusion on the practice in the GPRD might have had a fracture in the past. History of fractures was assessed, but was not a confounder in this study. We did not have the opportunity to assess whether the (prior) fracture was the result of highenergy trauma or whether epilepsy was induced by such trauma. Particularly among young male subjects, traffic accidents might account for a relatively large proportion of the fractures observed in the dataset. Also, it could be that patients with epilepsy and patients not having epilepsy differed with respect to the proportion of fractures induced by high-energy trauma, resulting in confounding. Still, the results of our analyses show that the difference in fracture incidence between the epilepsy and reference cohort was consistent in magnitude across age and sex strata.

We found that the fracture risk was higher among patients with a first epilepsy diagnosis during follow-up compared with patients having a first diagnosis of epilepsy in the past. This finding is probably not surprising, as it seems likely that newly diagnosed patients with epilepsy will be more prone to falls and fractures compared with patients having the disease for many years.

Several possible explanations exist for the increased fracture risk in patients with epilepsy. First, patients could have a fracture subsequent to a fall during an epileptic seizure. Second, long-term treatment with AEDs can result in a decrease in bone mineral density, thereby increasing the propensity for fracturing when falling. Third, it could be that patients with epilepsy have more risk factors for fractures compared with those without epilepsy, independent of AED use and seizure activity. This study was not designed to explore why the risk of fracture is increased in patients with epilepsy. The pathogenesis of fracture entails a complex interaction between bone mineral density on the one side and propensity to trauma on the other. Particularly in the highest age group, many other diseases and prescription drugs may have an effect on either of the two components relevant for a fracture to occur. Furthermore, we did not evaluate the effect of alcohol intake and smoking status, which might be confounders in this study and might explain part of the association observed.

In conclusion, this study showed that the incidence of fractures was nearly twofold increased among patients with a diagnosis of epilepsy compared with the general population. For the clinically most relevant fracture, hip and femur fracture, a threefold increased risk in elderly aged 50 years and older was found. Further study is necessary to elucidate whether this elevated risk is due to the disease, the use of AEDs, or both.

Acknowledgment: This study was funded by an unrestricted grant from GlaxoSmithKline, United Kingdom

\section{REFERENCES}

1. Chadwick D. Epilepsy. J Neurol Neurosurg Psychiatry 1994;57: 264-77.

2. Jacoby A, Buck D, Baker G, et al. Uptake and costs of care for epilepsy: findings from a U.K. regional study. Epilepsia 1998;39:776-86.

3. Kotsopoulos IA, van Merode T, Kessels FG, et al. Systematic review and meta-analysis of incidence studies of epilepsy and unprovoked seizures. Epilepsia 2002;43:1402-9.

4. Cummings SR, Melton LJ. Epidemiology and outcomes of osteoporotic fractures. Lancet 2002;359:1761-7.

5. Cummings SR, Nevitt MC. Falls. N Engl J Med 1994;331:8723.

6. Parrott S. The economic cost of hip fracture in the UK. York: University of York; 2000.

7. Persson HB, Alberts KA, Farahmand BY, Tomson T. Risk of extremity fractures in adult outpatients with epilepsy. Epilepsia 2002;43:768-72.

8. Vestergaard P, Tigaran S, Rejnmark L, et al. Fracture risk is increased in epilepsy. Acta Neurol Scand 1999;99:269-75.

9. Nilsson OS, Lindholm TS, Elmstedt E, et al. Fracture incidence and bone disease in epileptics receiving long-term anticonvulsant drug treatment. Arch Orthop Trauma Surg 1986;105:146-9.

10. Cummings SR, Nevitt MC, Browner WS, et al. Risk factors for hip fracture in white women: Study of Osteoporotic Fractures Research Group. N Engl J Med 1995;332:767-73.

11. Stephen LJ, McLellan AR, Harrison JH, et al. Bone density and antiepileptic drugs: a case-controlled study. Seizure 1999;8:33942.

12. Pack AM, Olarte LS, Morrell MJ, et al. Bone mineral density in an outpatient population receiving enzyme-inducing antiepileptic drugs. Epilepsy Behav 2003;4:169-74.

13. Sirven JI. Acute and chronic seizures in patients older than 60 years. Mayo Clin Proc 2001;76:175-83.

14. Walley T, Mantgani A. The UK General Practice Research Database. Lancet 1997;350:1097-9.

15. Van Staa TP, Abenhaim L, Cooper C, et al. The use of a large pharmacoepidemiologic database to study exposure to oral corticosteroids and risk of fractures: validation of study population and results. Pharmacoepidemiol Drug Saf 2000;9:359-66.

16. Van Staa TP, Abenhaim L. The quality of information recorded on a UK database of primary care records: a study of hospitalization due to hypoglycemia and other conditions. Pharmacoepidemiol Drug Saf 1994;3:32-4.

17. Van Staa TP, Dennison EM, Leufkens HG, Cooper C. Epidemiology of fractures in England and Wales. Bone 2001;29:517-22.

18. Annegers JF, Melton LJ 3rd, Sun CA, Hauser WA. Risk of agerelated fractures in patients with unprovoked seizures. Epilepsia 1989;30:348-55.

19. Mattson RH, Gidal BE. Fractures, epilepsy, and antiepileptic drugs Epilepsy Behav 2004;5(suppl 2):S36-40. 